\title{
Padrões de atividade física \\ de pessoas com transtornos mentais e de comportamento
}

\author{
Patterns of physical activity \\ of people with chronic mental and behavioral disorders
}

Angélica Nickel Adamoli ${ }^{1}$

$M$ ario Renato Azevedo ${ }^{2}$

\footnotetext{
${ }^{1}$ Programa de PósGraduação em Educação Física, Escola Superior de Educação Física, UFPEL. Rua Luiz deCamões 625, Bairro Tablada. 96055-630 Pelotas RS.

lilanickel@gmail.com

2 Escola Superior de Educação Física, UniversidadeFederal de Pelotas.
}

\begin{abstract}
Since physical activity (PA) is capable of improving both the quality of life and the prognosis for individuals with mental and behavioral disorders (MBD), the main purpose of this study was to analyze the PA patterns in individuals with $M B D$ frequenting a Psychosocial Care Center (PCC) in the city of Pelotas. The target population of this descriptive study consisted of individuals attended in any of the PCC s of Pelotas. The sample was sel ected from six PCCs and comprised 85 patients and their relatives. Themean age of thesample was 40.9 years (standard deviation 13.8). It was found that, in comparison with the general population, these individuals had a lower socioeconomic level and less schooling. The prevalence of leisure time physical activity was low. In addition, women tended to dedicate the greater part of their time to household activities. Men participated more in the PA offered by the PCC than women. Therefore, incorporation of PA in PCC seems to be a feasible initiative for supporting the treatment of these patients and would offer a unique opportunity for the patients to engage in supervised and structured PA programs.

Key words Physical activity, M ental health, Chronic mental and behavioral disorders
\end{abstract}

Resumo Considerando a atividadefísica (AF) como uma possibilidade viável de intervenção na melhora da qualidade de vida e prognóstico de indivíduos com transtornos mentais e de comportamento (TM C) , o objetivo desteestudo foi analisar o padrão de AF de pessoas com TM C, atendidas nos Centros de Atenção Psicossocial (CAPS) da cidade de Pelotas. Foi realizado um estudo descritivo sobre os padrões de AF deindivíduos com TMC. A populaçãoalvo deste estudo foi constituída pelas pessoas atendidas nos CAPS da cidade. A amostra foi composta por 85 usuários e seus familiares de seis CAPS da cidade. A média de idade dos usuários da amostra foi de 40,9 anos (DP 13,8). Verificou-se que esta população possui menor escolaridade emenor nível econômico secomparado à população em geral. Constatou-se baixa prevalência de AF de lazer, além de uma tendência, entre as mulheres, de utilizarem grande parte do seu tempo deAF em atividades domésticas. Entre as AF realizadas nos CAPS, os homens mostraram-se mais participativos do que as mulheres. Sendo assim, a prática de AF realizadas no atendimento dos CAPS parece ser uma iniciativa viável como uma das partes do tratamento destes indivíduos, oferecendo uma oportunidade única de prática física orientada e estruturada.

Palavras-chave Atividade física, Saúde mental, Transtornos mentais e de comportamento 


\section{Introdução}

As estimativas iniciais indicam que, atualmente, cerca de 450 milhões de pessoas sofrem transtornos mentais ou neurobiológicos ou, então, problemas psicossociais como os relacionados com o abuso do álcool e das drogas¹. Em termos globais, muitos indivíduos se transformam em vítimas por causa da sua doença e se convertem em alvos de estigma e discriminação ${ }^{1}$. Segundo a Organização M undial da Saúde (OM S), os transtornos mentais já representam quatro das dez principais causas de incapacitação em todo o mundo e esse crescente ônus vem a representar um custo enorme em termos de sofrimento humano, incapacidade e prejuízos econômicos ${ }^{1}$.

Os transtornos mentais e de comportamento (TMC) são uma série de distúrbios definidos pela Classificação Estatística Internacional de Doenças e Problemas de Saúde Correlatos $(\mathrm{CID}-10)^{2}$. Embora os sintomas variem consideravelmente, tais transtornos geralmente se caracterizam por uma combinação de idéias, emoções, comportamentos e relacionamentos anormais com outras pessoas. São exemplos a esquizofrenia, a depressão, o retardo mental e os transtornos devidos ao uso de substâncias psicoativas' ${ }^{1}$.

A relação saúde/doença mental éum processo particular de expressão das condições e do estilo de vida de uma sociedade, representando as diferentes qualidades do processo vital e as diferentes competências para enfrentar desafios, agressões, conflitos e mudanças, numa dupla e contraditória natureza: biológica e psicológica ${ }^{3}$. A importância da saúde mental é reconhecida pela OM S desde a sua origem e se reflete na atual definição de saúde como "um estado de completo bem-estar físico, mental e social"4.

É particularmente importante a compreensão dos determinantes do comportamento saudável devido ao papel que este desempenha na determinação do estado geral de saúde. As doenças não transmissíveis, como as cardiovasculares e o câncer, cobram um enorme tributo em saúde e vidas em todo o mundo. Muitas delas vinculam-se estreitamente a formas pouco saudáveis de comportamento, como o uso de álcool e tabaco, regime alimentar deficiente e vida sedentária. Além disso, o comportamento saudável de cada indivíduo depende muito da saúde mental da pessoa ${ }^{1}$.

A promoção da prática de atividades físicas (AF) nas populações tem ocupado lugar de destaque na agenda mundial de saúde pública. Isto de deve aos comprovados benefícios que a AF exerce para a prevenção de diversas doenças e manuten- ção da saúde, incluindo a mental ${ }^{5}$. Apesar disso, estudos mostram que o sedentarismo tem alcançado prevalências elevadas no mundo inteiro ${ }^{6-9}$.

$\mathrm{Na}$ busca de melhorias na assistência à saúde mental, movimentos sociais surgem e, com eles, se torna público o debate sobre a situação precária dos hospitais psiquiátricos que representavam 0 único recurso destinado aos usuários com TMC. N esse contexto, surgem em vários municípios os Centros de Atenção Psicossocial (CAPS), como dispositivos eficazes na diminuição de internações e na mudança do modelo assistencial. O CAPS é um serviço de saúde aberto e comunitário do SistemaÚ nico deSaúde(SUS). Eleéum lugar dereferência e tratamento para pessoas que sofrem de TM C, cuja severidadee/ou persistência justifiquem sua permanência num dispositivo de cuidado intensivo, comunitário, personalizado e promotor de vida ${ }^{10}$. 0 objetivo do CAPS é oferecer atendimento à população de sua área de abrangência, realizando acompanhamento clínico e a reinserção social dos usuários pelo acesso ao trabalho, lazer, exercício dos direitos civis e fortalecimento dos laços familiares e comunitários ${ }^{10}$.

Considerando a atividadefísica (AF) como uma possibilidade viável de intervenção na melhora da qualidade de vida eprognóstico deindivíduos com TM C, o objetivo deste estudo foi analisar o estilo de vida e o padrão de AF de pessoas com TMC atendidas nos CAPS da cidade de Pelotas.

\section{Metodologia}

Foi realizado um estudo descritivo sobre 0 estilo de vida e padrões de AF de indivíduos com TMC. A população-alvo do estudo foi constituída pelas pessoas atendidas nos CAPS da cidade de Pelotas, de ambos os sexos e que realizavam tratamento nas modalidades intensi vo (cinco dias na semana) e semi-intensivo (dois ou três dias na semana). 0 serviço de saúde mental conta com sete CAPS distribuídos nos bairros da cidade, sendo cada um responsável pelo atendimento de uma área territorial específica.

A amostra deste estudo foi composta de todos os indivíduos cujos familiares freqüentavam a reunião com a equipe multidisciplinar em seis CAPS da cidade. Em cada estabelecimento, uma visita foi realizada nos dias dessas reuniões. A pesquisadora responsável visitou todos os grupos nos CAPS da amostra, onde eram atendidos familiares ou responsáveis por pacientes em regime semi-intensivo eintensivo. Em função delimitações logísticas, um CAPS não foi incluído no estudo. 
Para esta pesquisa, elaborou-se um questionário para coletar as informações sobre os padrões deAF. N a ausência dequestionários específicos para essa população e, considerando a evidente dificuldadedecoletar informações através de entrevistas com os indivíduos com TMC, optou-se por elaborar um instrumento auto-administrado pelos familiares ou responsáveis dos usuários dos CAPS. Posteriormente, este instrumento foi submetido à análise de pesquisadores da área e testado através de um estudo-piloto com o objetivo de avaliar a aplicabilidade dos instrumentos elaborados e a logística da coleta de dados. Uma amostra de quinze usuários de dois CAPS da cidade, atendidos em regime não intensivo, foi selecionada para o estudo-piloto.

0 instrumento elaborado coletou informações sobre:

Dados de identificação do respondente: gênero, idade, escolaridade e parentesco da pessoa que concedeu a entrevista, ou seja, familiar ou responsável pelo usuário;

- Dados de identificação do usuário: idade, gê nero, escolaridade, estado civil enúmero defilhos;

- Dados domiciliares: classeeconômica dos participantes do estudo avaliada através do critério de classificação econômica daAssociação N acional de Empresas de Pesquisa (AN EP) ${ }^{11}$;

. Aspectos de saúde: tabagismo, internações em hospital psiquiátrico, consumo de medicamentos e doenças associadas ao TMC;

- AF do usuário: atividades físicas realizadas no trabalho, como meio de transporte, lazer e atividades domésticas;

. Atividades da vida diária (AVDs) e atividades instrumentais da vida diária (AIVDs): atividades da vida diária no ambientefamiliar ena comunidade, considerando o instrumento adaptado por $M$ arques';

. Ficha sobre a participação dos usuários nas AF do CAPS: tipo de AF praticada, número dedias por semana e tempo de realização da atividade, além da patologia do usuário. Este instrumento foi preenchido pelos professores de Educação Física do serviço.

Para a coleta dos dados foi solicitada autorização do coordenador do departamento de saúde mental do município, através da apresentação de um projeto de pesquisa. 0 estudo foi submetido à Comissão de Ética em Pesquisa da Faculdade de Medicina da Universidade Federal de Pelotas. A pós a aprovação, foi realizada uma reunião com os coordenadores dos CAPS para a apresentação da metodologia, estratégias adotadas para coleta dos dados e esclarecimentos sobre o estudo em questão e sua relevância.
Para a coleta de dados, durante as reuniões entre a equipe multidisciplinar e familiares e/ou responsáveis pelos usuários, foi solicitado o consentimento por escrito e entregue os questionários, realizando-se explicações sobre o preenchimento do mesmo e esclarecimentos de dúvidas.

Os dados foram digitados no programa EPI INFO. 0 tratamento estatístico do presente estudo foi realizado através de uma análise descritiva para caracterizar a população-alvo.

\section{Resultados}

A amostra foi composta por 85 usuários e seus familiares de seis CAPS da cidade de Pelotas, sendo $52,9 \%(n=45)$ pertencendo à modalidade intensiva e $47,1 \%(n=40)$, à semi-intensiva. Um dos CAPS ficou fora do estudo, pois no tempo previsto não foi possível fazer a coleta dos dados com os mesmos critérios utilizados para a pesquisa nos demais CAPS, o que modificaria as estratégias e andamento da pesquisa. 0 estudo atingiu a participação de $15 \%$ dos usuários dos seis CAPS investigados. A participação em todos os CAPS variou de $10,6 \%$ a $24,4 \%$.

Entre os familiares ou responsáveis dos usuários dos CAPS, houve predominância de mulheres $(n=67 ; 78,8 \%)$ emães $(n=35 ; 41,7 \%)$. A idade mé dia dos familiares ou responsáveis foi de 50,3 anos (DP 14,8) e, segundo a escolaridade, aproximadamente metade $(50,6 \%)$ estudou até, no máximo, a quarta série do ensino fundamental.

Com relação ao sexo, a maioria da amostra era de usuários do sexo masculino $(62,3 \% ; n=53)$. A média de idade dos usuários da amostra foi de 40,9 (DP 13,8). A escolaridademédia foi de 3,8 anos de estudo (DP 3,3). Os transtornos mentais e de comportamento apresentados na amostra foram: esquizofrenia $(n=25)$, retardo mental $(n=20)$, de pressão $(n=17)$, transtornos por uso de álcool $(n=9)$, transtornos orgânicos $(n=8)$, transtornos de personalidade $(n=2)$, psicose $(n=2)$, transtornos de ansiedade $(n=1)$ e transtornos por uso de $\operatorname{drogas}(n=1)$.

Outras doenças associadas, referidas pelos indivíduos da amostra, foram: hipertensão $(n=25$; $29,4 \%$ ), colesterol elevado ( $n=12 ; 14,1 \%$ ), asma el ou bronquite $(n=12 ; 14,1 \%)$, problemas cardíacos $(n=7 ; 8,2 \%)$ e diabetes $(n=6 ; 7,1 \%)$. Entre os indivíduos que fumam todos os dias ( $n=28)$, a média de cigarros por dia foi de 24,8 (DP 21,2). A penas seis indivíduos $(7,1 \%)$ relataram trabalhar de forma remunerada ou voluntária. As atividades profissionais citadas foram: reciclagem de material 
$(n=2)$, servente de pedreiro $(n=2)$, artesanato $(n=1)$ e comerciante $(n=1)$. A Tabela 1 traz a descrição dos usuários da amostra segundo variáveis demográficas, socioeconômicas e de saúde.

Tabela 1. Descrição dos usuários da amostra segundo variáveis demográficas, socioeconômicas e de saúde.

\begin{tabular}{|c|c|c|}
\hline Variável & $\mathrm{N}$ & $\%$ \\
\hline \multicolumn{3}{|l|}{ Sexo } \\
\hline M asculino & 53 & 62,3 \\
\hline Feminino & 32 & 37,7 \\
\hline \multicolumn{3}{|l|}{ I dade (anos completos) } \\
\hline Até 35 & 33 & 39,3 \\
\hline $36-49$ & 28 & 33,3 \\
\hline 50 ou mais & 23 & 27,4 \\
\hline \multicolumn{3}{|l|}{ Nível econômico } \\
\hline$B$ & 5 & 6,02 \\
\hline $\mathrm{C}$ & 20 & 3,8 \\
\hline $\mathrm{D}$ & 51 & 60,7 \\
\hline$E$ & 8 & 9,5 \\
\hline \multicolumn{3}{|l|}{ Escolaridade (anos de estudo) } \\
\hline 0 & 19 & 22,6 \\
\hline $1-4$ & 33 & 39,3 \\
\hline $5-8$ & 23 & 27,4 \\
\hline 9 ou mais & 9 & 10,7 \\
\hline \multicolumn{3}{|l|}{ Situação conjugal } \\
\hline Casado ou c/ companheiro & 25 & 29,4 \\
\hline Solteiro ou s/ companheiro & 51 & 60,0 \\
\hline Divorciado & 7 & 8,2 \\
\hline Viúvo & 2 & 2,4 \\
\hline \multicolumn{3}{|l|}{ Número de filhos } \\
\hline 0 & 45 & 53,6 \\
\hline 1 & 10 & 11,9 \\
\hline 2 & 12 & 14,3 \\
\hline 3 ou mais & 17 & 21,2 \\
\hline \multicolumn{3}{|l|}{ Tabagismo } \\
\hline Nunca fumou & 43 & 50,0 \\
\hline Ex-fumante & 10 & 11,9 \\
\hline Fumante & 32 & 38,1 \\
\hline \multicolumn{3}{|l|}{ Internação psiquiátrica } \\
\hline Não & 36 & 42,3 \\
\hline Sim & 49 & 57,7 \\
\hline
\end{tabular}

Com relação às atividades da vida diária, a prevalência de indivíduos que realizam suas atividades com dificuldade ou que não consegue fazê-las deforma individual variou de 2,4\% (alimentar-se) a 12,9\% (vestir-se, realizar higiene pessoal e arrumar-se/pentear-se).

Entre as atividades instrumentais da vida diária, cerca de 2/3 da amostra demonstraram, segundo relato do familiar ou responsável, condições deutilizar transporte coletivo (68,2\%) ou usar o tel efone $(67,4 \%)$. A Tabela 2 traz a descrição completa da amostra segundo o grau de independência para as atividades da vida diária.

Com relação ao meio de transporte utilizado pelos usuários para se deslocar para o trabalho $(n=6)$, dois utilizavam a bicicleta e um a caminhada. 0 meio de transporte para os CAPS mais utilizado foi o ônibus ( $n=42 ; 49,4 \%)$. Entre os meios dedeslocamento ativos, 36 indivíduos $(42,4 \%)$ utilizavam a caminhada e cinco $(5,9 \%)$ a bicicleta. Ainda, uma pessoa utilizou a motocicleta como meio de transporte para o CAPS. Aproximadamente $70 \%(n=57)$ dos usuários da amostra utilizavam a bicicleta $(n=14 ; 17,1 \%)$ ou caminhada $(n=43 ; 52,4 \%)$ como meio de deslocamento para outros lugares, como passeios e compras.

Considerando o tempo total utilizado com caminhadas nos deslocamentos para o trabalho, CAPS e outros lugares, a média semanal em minutos entre os indivíduos que realizavam al guma caminhada como meio de deslocamento $(n=56 ; 66 \%)$ foi de 146 minutos (DP 106).

A utilização da bicicleta como meio de transporte para o trabal ho, CAPS ou outros lugares foi relatada por dezesseis indivíduos (18,8\%) e, entre estes, a média semanal em minutos de deslocamento com a bicicleta foi 145 minutos (DP 130).

Tabela 2. Descrição da amostra segundo o grau de independência para as atividades instrumentais da vida diária.

\begin{tabular}{lccc}
\hline \multirow{2}{*}{\multicolumn{1}{c}{ Atividade }} & \multicolumn{3}{c}{ Grau de independência } \\
\cline { 2 - 4 } & Consegue & Consegue com dificuldade & Não consegue \\
\hline Lidar com dinheiro & 61,1 & 11,8 & 27,1 \\
U sar transporte coletivo & 68,2 & 16,5 & 15,3 \\
Fazer compras & 52,9 & 16,5 & 30,6 \\
Cuidar da saúde/medicar-se & 42,4 & 28,2 & 29,4 \\
Comunicar-se com as pessoas & 88,2 & 5,9 & 5,9 \\
U sar o telefone & 67,4 & 12,1 & 20,5 \\
\hline
\end{tabular}


Dentre as AF realizadas no tempo de lazer dos indivíduos, andar de bicicleta (8,5\%) e caminhadas $(7,1 \%)$ foram as atividades mais citadas. A Tabela 3 traz a descrição completa das AF realizadasno período delazer entre os usuários dos CAPS.

Tabela 3. Descrição das atividades físicas de lazer dos usuários dos CAPS.

\begin{tabular}{llrr}
\hline \multicolumn{1}{c}{ Atividade } & N & $\%$ & $\begin{array}{c}\text { M inutos por semana } \\
\text { Média }(D P)^{*}\end{array}$ \\
\hline Andar de bicicleta & 8 & 8,5 & $171,3(144,0)$ \\
Caminhada & 6 & 7,1 & $95,0(48,1)$ \\
Futebol & 3 & 3,5 & $140,0(138,6)$ \\
Dança & 2 & 2,4 & $30(0,0)$ \\
Ginástica & 2 & 2,4 & $120(84,9)$ \\
Basquete & 1 & 1,2 & $180(0,0)$ \\
\hline
\end{tabular}

* Considerando somenteosindivíduos que realizam tais atividades

Tabela 4. Descrição das atividades físicas realizadas nos CAPS.

\begin{tabular}{lccc}
\hline \multicolumn{1}{c}{ Atividade } & N & $\%$ & $\begin{array}{c}\text { Minutos por semana } \\
\text { Média (DP)* }\end{array}$ \\
\hline Futebol & 19 & 22,4 & $54,2(12,2)$ \\
Recreação & 19 & 22,4 & $53,4(32,8)$ \\
Ginástica & 29 & 34,1 & $28,6(15,5)$ \\
Caminhada & 12 & 14,1 & $30,4(12,0)$ \\
Dança & 6 & 7,1 & $83,3(16,3)$ \\
Atividades aquáticas & 6 & 7,1 & $60,0(0,0)$ \\
Oficinas de esportes** & 5 & 5,9 & $28,0(16,4)$ \\
\hline
\end{tabular}

* Considerando somente os indivíduos que realizam tais atividades.

** Aulas que contemplam diversas modalidades esportivas.
Entre as AF realizadas nos CAPS, orientadas por professores de Educação Física, as aulas de ginástica (34,1\%), futebol $(22,4 \%)$ e recreação $(22,4 \%)$ foram as mais citadas. A Tabela 4 traz a descrição completa das AF realizadas nos CAPS ea média semanal em minutos que os indivíduos se envolvem em cada atividade.

Com relação às $A F$ realizadas em casa, varrer $(57,5 \%)$, lavar a louça (55,3\%), lavar roupa (27,0\%) e cuidar do jardim (18,8\%) foram as mais citadas. Entre os indivíduos que se envolviam com tais atividades, a média semanal em minutos para cada tipo de atividades variou de 35 (levantar ou carregar objetos pesados) a 160 (lavar roupas à mão).

A Figura 1 traz o gráfico da prevalência de não envolvimento com AF nos diferentes domínios investigados, estratificado por sexo. Enquanto os homens demonstraram um menor envolvimento com AF domésticas, as mulheres evidenciaram uma grandeinatividadefísica no atendimento dos CAPS.

Em relação à contribuição de cada domínio sobre o tempo total de envolvimento com AF dos indivíduos do sexo masculino, constatou-seo predomínio deatividades domésticas (39,4\%) secomparado às demais: deslocamento $(34,8 \%)$, CAPS $(15,7 \%)$ e lazer $(10,1 \%)$. Já entre as mulheres, as atividades domésticas representaram $62,9 \%$ do tempo total em atividades físicas semanais, mostrando índice bem superior aos demais domínios: deslocamento (24,1\%), CAPS $(6,9 \%)$ elazer (6,1\%).

\section{Discussão}

Considerando os comprovados benefícios da AF para saúde como um todo, os resultados apresentados neste estudo trazem uma importante con-

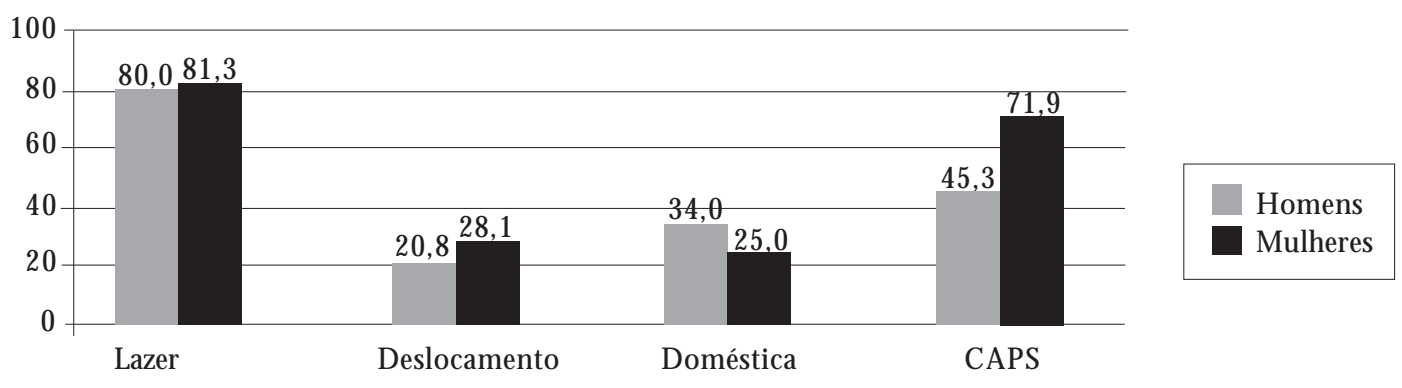

Figura 1. Prevalência de não envolvimento com atividades físicas nos diferentes domínios investigados, estratificado por sexo. 
tribuição para 0 entendimento e futuras linhas de ações no que diz respeito, especialmente, ao conhecimento sobre os padrões de AF de indivíduos com TMC.

Nossos achados reforçam a necessidade de aperfeiçoamento no serviço oferecido nos CAPS, tendo em vista o quadro desfavorável apresentado em relação à prática de AF. Entretanto, cabe destacar que 0 atendimento às pessoas com TM C é amplo, abordando desde cuidados clínicos a atividades de reabilitação psicossocial, visando a substituir o modelo hospitalocêntrico, evitando as internações e favorecendo o exercício da cidadania e da inclusão social dos usuários e de suas famílias na comunidade ${ }^{10}$. Esteserviço conta com uma equipe multidisciplinar na qual, além do profissional de Educação Física, atuam psiquiatras, médicos clínicos, psicólogos, assistentes sociais, artistas plásticos, músicos, enfermeiros, auxiliares deenfermagem, terapeutas ocupacionais e artesãos.

No que diz respeito à metodologia empregada nesteestudo, al gumas consider ações devem ser feitas para avaliar a extrapolação destes resultados. Inicialmente, em relação à prevalência maior de homens em nossa amostra (62,4\%) se comparado às mulheres (37,7\%), devemos destacar que a realidade quanto ao gênero desta população compre ende uma maioria de mulheres (54,6\%). Como a logística do estudo previa a partici pação dos indivíduos cujos familiares participassem das reuniões mensais em cada CAPS, provavelmente há algum fator determinante para que os responsáveis pelas mulheres não compareçam com a mesma assiduidade que os familiares dos homens. Tratando-se de esposos, por exemplo, possivelmente estes tenham que trabal har nos horários de reuniões. De forma a minimizar o efeito desta discre pância, a maioria das análises foi conduzida de forma estratificada quanto ao gênero.

0 mesmo acontece quanto à modalidade de atendimento, em quea população real semi-intensiva $(63,7 \%)$ supera a modalidade intensiva $(36,3 \%)$. Na amostra do presente estudo, a predominância de indivíduos do sistema intensivo pode ser resultante da necessidade de uma maior participação dos responsáveis ou familiares junto ao serviço de saúde no tratamento do usuário. Para este caso, após analisar mais detalhadamente os padrões de AF entre indivíduos das duas modalidades de atendimento, não se constatou grandes diferenças entre os grupos.

Com relação à amostra estudada, ficou evidente a caracterização de uma população de menor nível econômico e de menor escolaridade se comparado à população em geral ${ }^{6}$. Esta constatação se fez importante, no primeiro momento, para a aplicação do instrumento de coleta de dados. Neste ponto, devemos destacar a logística para esta tarefa, pois apesar do questionário ser auto-administrado pelo familiar ou responsável do usuário, as diversas dúvidas foram esclarecidas no momento da coleta. M as, mesmo assim, éperfeitamente plausível esperar algum erro de informação sobre alguns hábitos de seus parentes usuários dos CAPS.

Cabe destacar que a opção por coletar as informações com os familiares se deu pela estimativa de uma parcela significativa da população-alvo com retardo mental, confirmada no estudo $(n=20)$, que possivelmente não responderia com precisão a uma entrevista.

As condições socioeconômicas desfavoráveis têm sido constantemente associadas a comportamentos menos saudáveis. Por exemplo, indivíduos de classes econômicas mais baixas ou de menor escolaridade apresentam as maiores prevalências detabagismo $0^{13}$ eestilo devida sedentário ${ }^{14}$. Em relação à prática de $\mathrm{AF}$, cabe reconhecer que se trata de um fenômeno multicausal, dependente de fatores econômicos, demográficos, sociais, emocionais, biológicos e do meio ambiente ${ }^{15,16}$. No caso específico de nossa população, os determinantes socioeconômicos desfavoráveis e as diferentes características da doença mental, como sintomas de cada tipo deTM C e efeitos colaterais das medicações, podem influenciar de forma negativa a prática de AF.

Um fator de extrema importância à saúde constatado no presente estudo foi a elevada prevalência de tabagismo se comparado à população em geral. Em se tratando de uma população especial, não se pode deixar de registrar a associação entre TM C e tabagismo, em queum exemplo importante éa esquizofrenia. A maioria dos esquizofrênicos são fumantes, têm uma dieta pobre, apresentando sobrepeso ou obesidade ${ }^{17}$. N a amostra de nosso estudo, observou-se que a maior parcela de usuários que fumam diariamente e em maior quantidade está entre os esquizofrênicos (45,8\%) se comparado às outras doenças $(35,0 \%)$ (dados não mostrados). Em um estudo realizado com pacientes esquizofrênicos, 71 (70\%) eram fumantes, 14 (14\%), ex-fumantes e 14 (14\%) nunca tinham fumado ${ }^{18}$.

$\mathrm{Na}$ nossa amostra, dezessete indivíduos $(20,0 \%)$ foram diagnosticados como sendo depressivos. A depressão grave é atualmente a principal causa de incapacitação em todo o mundo e situase em quarto lugar entre as dez principais causas da carga patológica mundial. Se estiverem corretas as projeções, caberá à depressão nos próximos vinte anos ser a segunda das princi pais causas da carga mundial de doenças ${ }^{1}$. 
A promoção de um estilo de vida fisicamente ativo é um objetivo importante da saúde pública atual ${ }^{18}$. 0 comportamento sedentário é uma das causas principais de morbidade e mortalidade ${ }^{19}$. Pessoas com TMC são significativamente menos ativas do que a população geral, devido a diversos fatores associados ${ }^{20}$. De acordo com o estudo de Lawrence, citado em Richardson et al. ${ }^{20}$, em média, populações com doenças mentais sérias morrem dez a quinzeanos mais cedo quea população geral. Embora alguns dos motivos da mortalidade seja devido ao suicídio e à morte acidental, a doença isquêmica do coração é uma causa comum de mortalidade adicional nesta população ${ }^{20}$.

Algumas pesquisas envolven do AF eTM $C$ vêm sendo realizadas, evidenciando alguns aspectos positivos desta relação. Estudos descrevem que a AF pode ser uma medida eficaz para o tratamento e prevenção de doenças psiquiátricas, tais como a depressão ${ }^{21-27}$ e ansiedade ${ }^{18,25,27-29}$.

Evidências também apontam para uma relação positiva da AF com a qualidade de vida ${ }^{25,30} \mathrm{e}$ vitalidade dos participantes ${ }^{25}$. Além disso, a AF parece estar associada com melhoras no bem-es$\operatorname{tar}^{23,25}$, auto-estima, auto-eficácia, encorajando e gerando pensamentos, sentimentos positivos que servem para contrariar o humor negativo ${ }^{24,26}$. No entanto, o conhecimento sobre a AF e humor é ainda limitado, não sendo possível definir a relação de causa e efeito ou descrever em detalhes os mecanismos psicológicos e fisiológicos subjacentes a esta associaçãa ${ }^{28}$.

Baseando-se na teoria das inter-relações extensamente reconhecidas entre a saúde física e mental, Rohrer et al. ${ }^{31}$ testaram a hipótese de que as pessoas que relatavam estilos de vida saudáveis também relatariam uma saúde mental melhor, investigando, entre outros fatores, a AF. Os autores concluíram que estratégias da saúde pública projetadas para melhorar o estilo de vida conduzirão às melhorias na saúde mental, bem como da saúde física. A saúdemental boafoi associadaindependentementecom a $A F^{31}$. Ainda nesteestudo, os resultados evidenciaram que as pessoas que se exercitaram cinco ou mais dias na semana eram muito mais prováveis de relatar a saúde mental boa do que as pessoas que não relataram nenhum exercício, e até mesmo um dia de exercício tornava-se um tanto protetor para a saúde mental do que nenhum ${ }^{31}$.

0 presente estudo procurou investigar os padrões de AF dos usuários dos CAPS, analisando suas práticas em cada um dos domínios do seu cotidiano. Com relação às AF realizadas no deslo- camento, estas se apresentaram significativamente elevadas no total das AF cotidianas dos usuários, tanto para o gênero masculino (34,8\%), quanto para o feminino (24,1\%). No gênero feminino, estas AF só perdem em tempo para as domésticas. Estes resultados podem ser explicados pelo fato da maioria dos usuários residirem próximos deste serviço de saúde, o que favorece o deslocamento de forma ativa. No entanto, não se pode descartar o baixo poder aquisitivo da população atendida no CAPS como um limitador do uso de meios de transporte pagos. Alguns usuários têm a possibilidade de utilizar o transporte coletivo gratuitamente, pelo fato de terem recebido aposentadoria antecipada por "incapacidade" para realizar atividades laborais e se auto-sustentar, embora este benefício atinja uma minoria.

Com relação às atividades de lazer, se constatou um quadro de baixa prevalência de AF. De modo geral, a prática de atividades físicas no período de lazer é baixa na população ${ }^{14,32}$. Para esse grupo em especial, a dificuldade do indivíduo em participar de grupos sociais devido à patologia e sua evidente falta de interação com a sociedade nos momentos de lazer pode ser um fator determinante.

A média semanal de tempo gasto em AF pelos homens foi maior que para as mulheres no lazer. Tal achado também aparece no estudo de Silveira e Duarte ${ }^{23}$, quando avaliaram pacientes depressivos. Além disso, os dados mostram ainda uma tendência entre as mulheres de ocuparem grande parte do tempo realizando atividades domésticas, diminuindo assim o tempo disponível para o lazer. Esse é um fato que, possivelmente, possa explicar o maior tempo gasto de AF no lazer entre os homen ${ }^{23}$.

Entre as AF realizadas nos CAPS, os homens mostraram-se mais participativos do que as muIheres. Esta diferença pode ser explicada pelo tempo com que os homens se envolvem com atividades como o futebol ou outros esportes, enquanto as mulheres têm participação em oficinas mais passivas, como bordado, pintura, crochê e AF leves, como alongamentos, caminhadas curtas e relaxamento. Considerando a relevância para a saúde, a participação efetiva destas pessoas em programas de AF orientadas que obedeçam aos princípios básicos de um treinamento físico e, principalmente, diante da constatação de que para a grande mai oria é no CAPS a única oportunidade de envolvimento com uma AF estruturada, é preocupante a baixa adesão nestas atividades, especialmente entre as mulheres. 


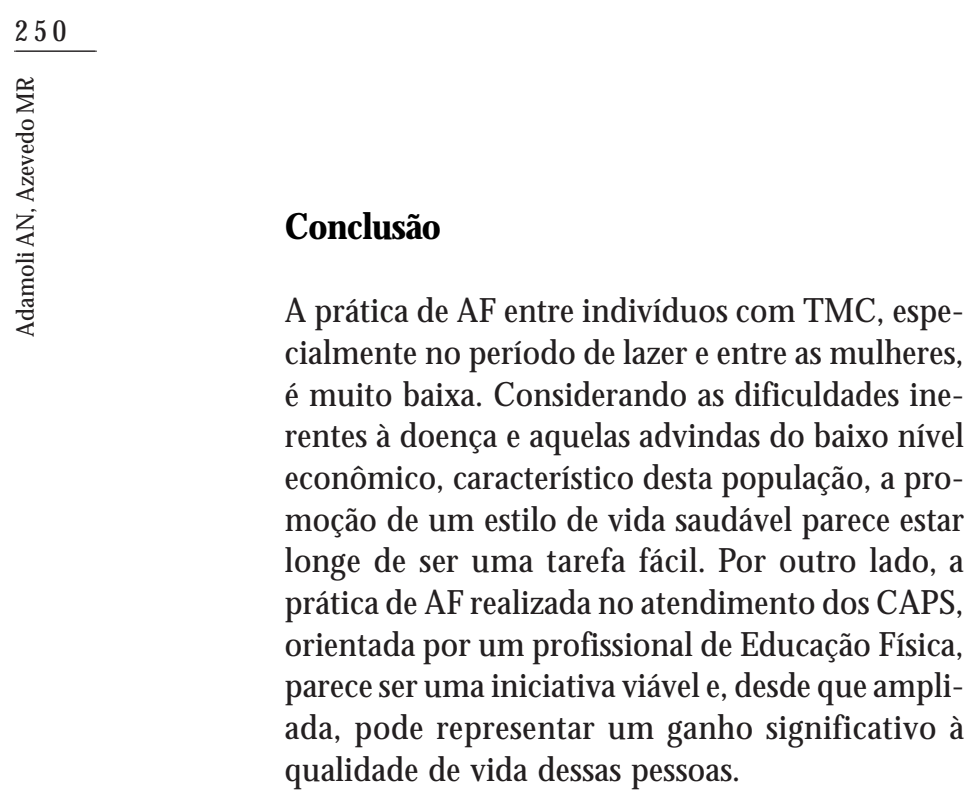

Colaboradores

AN Adamoli foi responsável por todas as fases do estudo, desde a elaboração do projeto à redação do artigo final; M R Azevedo Júnior participou da elaboração do projeto, análise dos dados e redação do artigo final.

\section{Referências}

1. Organização M undial de Saúde, Organização PanAmericana de Saúde. Relatório sobre a Saúde no mundo 2001: Saúde mental: nova concepção, nova esperança. Geneva: OM S; 2001.

2. Organização Mundial da Saúde. Classificação dos Transtornos M entais e de Comportamento da CID-10: Descrições Clínicas e Características Diagnósticas. Porto Alegre: Artes M édicas; 1993.

3. Sampaio JC. Qualidade de vida no âmbito da saúde mental. In: Roeder MA. Atividade física, saúde mental e qualidade de vida. Rio de Janeiro: Shape; 2003. p. 29-52.

4. Pereira MG. Epidemiologia: teoria e prática. Rio de Janeiro: Guanabara Koogan; 1995.

5. Bauman AE. Updating the evidence that physical activity is good for health: an epidemiological review 2000-2003. J Sci M ed Sport 2004; 7(1 Suppl):6-19.

6. Hallal PC, Victora CG, Wells JC, Lima RC. Physical inactivity: prevalence and associated variables in Brazilian adults. M ed Sci Sports Exerc 2003; 35(11):18941900.

7. Matsudo $S M, M$ atsudo VKR, Araújo $T$, Andrade $D$, Andrade E, Oliveira L, Braggion G. Nível de atividade física da população de São Paulo: análise de acordo com o gênero, idade, nível sócio-econômico, distribuição geográfica e de conhecimento. $\mathrm{R}$ Bras $\mathrm{Ci}$ M ov 2002; 10(4):41-50.

8. Gomez LF, Mateus JC, Cabrera G. Leisure-time physical activity among women in a neighbourhood in Bogota, Colombia: prevalence and socio-demographic correlates. Cad Saúde Pública 2004; 20(4):1103-1109.

9. Burton NW, Turrell G. Occupation, hours worked, and leisure-time physical activity. Prev M ed 2000; 31(6):673-681.

10. Brasil. M inistério da Saúde. Secretaria de Atenção à Saúde. Departamento de Ações Programáticas Estratégicas. Saúde mental no SU S: os centros de atenção psicossocial. Brasília: Ministério da Saúde; 2004.

11. Associação Nacional de Empresas de Pesquisa. Crité rio de classificação econômica Brasil. São Paulo: Associação Nacional de Empresas de Pesquisa; 2000. 
12. Marques AC. Q ualidade de vida de pessoas com síndrome de Down, maiores de 40 anos, no estado de Santa Catarina [dissertação]. Florianópolis (SC): Universidade Federal de Educação Física; 2000.

13. Moreira LB, Fuchs FD, M oraes RS, Bredemeir M, Cardozo S. Prevalence of smoking and associated factors in a metropolitan area in the southern region of Brazil. Rev. Saude Pública 1995; 29(1):46-51.

14. Dias-da-Costa JS, Hallal PC, Wells JC, Daltoe T, Fuchs SC, Menezes AM, Olinto MT. Epidemiology of leisure-time physical activity: a population-based study in southern Brazil. Cad Saúde Pública 2005; 21(1):275282.

15. Iso-Ahola S. Motivational foundations of leisure. In: Jackson EL, Burton TL, editors. Leisure Studies: Prospects for the XXI century. State College, PA: Venture Publishing; 1999. p. 35-51.

16. Trost SG, O wen N, Bauman AE, Sallis JF, Brown W. Correlates of adults' participation in physical activity: review and update. M ed Sci Sports Exerc. 2002; 34:1996-2001.

17. M CCreadie RG, Scottish Schizophrenia Lifestyle Group. Diet, smoking and cardiovascular risk in people with schizophrenia: descriptive study. Br J Psychiatry 2003; 183:534-539.

18. World Health Organization. Global strategy on diet, physical activity and health. Geneva: WHO; 2004.

19. US Department of Health and Human Services. Physical Activity and Health: A Report of the Surgeon General. Atlanta: Center for Disease Control and Prevention/National Center for Chronic Disease Prevention and Health Promotion; 1996.

20. Richardson CR, Faulkner G, M CD evitt J, Skrinar GS, Hutchinson DS, Piette JD. Integrating physical activity into mental health services for persons with serious mental illness. Psychiatr Serv 2005; 56(3):324-331.

21. Knechtle B. Influence of physical activity on mental well-being and psychiatric disorders. Schweiz Rundsch M ed Prax 2004; 93(35):1403-1411.

22. Dunn AL, Trivedi M H, Kampert JB, Clark CG, Chambliss HO. Exercise Treatment for Depression: Efficacy and Dose Response. A J Prev M ed 2005; 28(1):1-8.
23. Silveira LD, Duarte M FS. Níveis de depressão, hábitos e aderência a programas de atividades físicas de pessoas diagnosticadas com transtorno depressivo. Rev Bras Cine Des H um 2004; 6(2):36-44.

24. Craft LL, Perna FM. The Benefits of Exercise for the Clinically Depressed. Prim Care Companion J Clin Psychiatry 2004; 6(3):104-111.

25. Abu-Omar K, Rutten A, Lehtinen V. Mental health and physical activity in the European Union. Soz Praventivmed 2004; 49(5):301-309.

26. Roeder MA. Benefícios da atividade física para pessoas com transtornos mentais. Rev Bras Ativ Fís e Saúde 1999; 4(2):62-76.

27. Cheik NC, Reis IT, Heredia RG, Ventura ML, Tufik S, Antunes HKM , Mello MT. Efeitos do exercício físico e da atividade física na depressão e ansiedade em indivíduos idosos. R Bras Ci Mov 2003: 11(2):41-47.

28. Peluso MA, Andrade LH. Physical activity and mental health: the association between exercise and mood. Clinics 2005; 60(1):61-70.

29. Paluska AS, Schwenk TL. Physical activity and mental health: current concepts. Sports M ed 2000; 29(3):167180.

30. Schmitz N, Kruse J, Kugler J. The association between physical exercises and health-related quality of life in subjects with mental disorders: results from a cross-sectional survey. Prev. Med 2004; 39(6):12001207.

31. Rohrer JE, Pierce JR Jr, Blackburn C. Lifestyle and mental health. Prev Med 2005; 40(4):438-443.

32. Barros MV, Nahas MV. [Health risk behaviors, health status self-assessment and stress perception among industrial workers]. Rev Saude Pública. 35:554-563, 2001.

Artigo apresentado em 21/06/2006

Aprovado em 06/10/2006

Versão final apresentada em 19/03/2007 